\title{
LA JUSTICIA GRATUITA EN ESPAÑA: APROXIMACIÓN A UN ANÁLISIS CUANTITATIVO
}

\author{
José María Aguilar GonZÁLEZ \\ Profesor en la Universidad Complutense de Madrid \\ Profesor asociado en la Universidad Internacional de La Rioja \\ jm.aguilar@der.ucm.es
}

\begin{abstract}
RESUMEN
El artículo analiza brevemente el sistema de justicia gratuita tanto en España como en varios países de nuestro entorno y realiza una aproximación cuantitativa al coste que supone para las Administraciones Públicas la provisión de este servicio.
\end{abstract}

Palabras clave: justicia gratuita, bienes públicos, presupuesto público, análisis económico.

\section{ABSTRACT}

The article briefly analyses the legal aid system as much in Spain as in several countries around it and makes a quantitative approach of the expenses that such a service brings upon the public administrations.

Keywords: Legal Aid, Public Goods, Public Budget, Economic Analysis.

\section{ZUSAMMENFASSUNG}

Der Artikel untersucht knapp das System der kostenfreien Justiz in Spanien und in verschiedenen umliegenden Staaten und fübrt eine quantitative Untersuchung dazu durch, was die Bereitstellung dieses Service den einzelnen öffentlichen Verwaltungen an Kosten verursacht.

Schlüsselwörter: Kostenlose Justiz, öffentliche Güter, öffentlicher Haushalt, Kostenanalyse.

SUMARIO: I. INTRODUCCIÓN.-II. LA JUSTICIA GRATUITA.-1. Concepto, ámbito y contenido.-2. Justificación.-III. LA JUSTICIA GRATUITA EN LA UNIÓN EUROPEA.-1. Bélgica.-2. Alemania.-3. Inglaterra y País de Gales.-4. Francia.-IV. LA JUSTICIA GRATUITA EN ESPANA.-V. CONCLUSIONES.-VI. BIBLIOGRAFÍA. 


\section{INTRODUCCIÓN}

La reciente entrada en vigor de la Ley de Tasas ${ }^{1}$ ha avivado el debate sobre el derecho a la tutela judicial efectiva que, como señala el preámbulo de la Ley 10/2012, no debe ser confundido con el derecho a la justicia gratuita. La implementación de políticas que actúan directamente sobre la demanda de los servicios de justicia, intentando, entre otras cuestiones, reducir una demanda considerada «excesiva» ${ }^{2}$, tiene importantes efectos sobre el sistema de justicia gratuita, ya que suponen, en el caso de la eliminación de la subvención de las tasas judiciales, un aumento del coste del litigio. De este modo los grupos de renta baja se pueden encontrar en una situación de falta de recursos para litigar. El objeto de este artículo no es analizar el sistema de tasas judiciales o estudiar los incentivos o desincentivos que genera el sistema, sino aproximarse al coste que para el sector público tiene el sistema de justicia gratuita. Dado que las nuevas tasas suponen un aumento del coste del litigio, ¿qué alcance tiene el derecho de justicia gratuita?, ¿ ‘ómo se implementa en España y en otros países de nuestro entorno?, ¿'es posible conocer el esfuerzo que supone para el Estado el sistema de justicia gratuita? El artículo comienza, en su primera parte, analizando el concepto, ámbito, contenido y justificación del beneficio de justicia gratuita. En la segunda parte se lleva a cabo un análisis comparativo de los diferentes sistemas de servicios de justicia gratuita en varios países de nuestro entorno (Bélgica, Alemania, Inglaterra y País de Gales y Francia) y se ofrecen datos relacionados con el presupuesto anual destinado a la justicia gratuita en el ámbito de la Unión Europea. A continuación se analiza brevemente el sistema en España, incluyendo una sencilla aproximación cuantitativa del coste del sistema en territorios donde no se han transferido las competencias en materia de justicia gratuita.

${ }^{1}$ Ley 10/2012, de 20 de noviembre, por la que se regulan determinadas tasas en el ámbito de la Administración de Justicia y del Instituto Nacional de Toxicología y Ciencias Forenses (BOE, núm. 280, de 21 de noviembre de 2012).

2 Santos Pastor (1993), refiriéndose al papel «racionador» de los precios, señala que: «En general, si el disfrute de un beneficio por un usuario exigen un coste, que el usuario pague un precio para compensar ese coste es socialmente "adecuado": desincentiva un consumo excesivo y evita la injusticia de que otros paguen por ello». 


\section{LA JUSTICIA GRATUITA}

\section{Concepto, ámbito y contenido}

La Constitución Española, en su art. 24, establece que «todas las personas tienen derecho a obtener la tutela efectiva de los jueces y tribunales en el ejercicio de sus derechos e intereses legítimos, sin que, en ningún caso, pueda producirse indefensión». El ejercicio de este derecho a la tutela efectiva de los jueces y tribunales tiene aparejados una serie de costes para los individuos que, en caso de no poder sufragarlos, los situarían en una situación de indefensión. Como el propio artículo considera que en ningún caso puede producirse indefensión, la falta de recursos económicos para litigar no puede ser causa de ésta, y así, el art. 119 de la Constitución establece que la justicia será gratuita cuando lo disponga la ley y, en todo caso, «respecto de quienes acrediten insuficiencia de recursos para litigar». Este derecho es desarrollado por la Ley 1/1996, de 10 de enero, de Asistencia Jurídica Gratuita, y otra serie de normas tanto estatales como autonómicas ${ }^{3}$. El derecho

${ }^{3}$ Las principales normas de ámbito estatal que regulan la asistencia jurídica gratuita son las siguientes: Ley 1/1996 de Asistencia Jurídica Gratuita (BOE de 12 de enero de 1996); Real Decreto 996/2003, de 25 de julio, por el que se aprueba el Reglamento de Asistencia Jurídica Gratuita (BOE, núm. 188, de 7 de agosto de 2003); Real Decreto 1455/2005, de 2 de diciembre, por el que se modifica el Reglamento de Asistencia Jurídica Gratuita aprobado por el Real Decreto 996/2003, de 25 de julio; Ley 16/2005, de 18 de julio, por la que se modifica la Ley 1/1996, de 10 de enero, de Asistencia Jurídica Gratuita, para regular las especialidades de los litigios transfronterizos civiles y mercantiles en la Unión Europea, y Orden de 3 de junio de 1997, por la que se establecen los requisitos generales mínimos de formación y especialización necesarios para prestar los servicios de asistencia jurídica gratuita. En cuanto a las normas autonómicas, no todas las Comunidades Autónomas han aprobado normas a este respecto, sólo Galicia, Cantabria, Andalucía, Madrid, Cataluña, Valencia, Canarias, Aragón y Navarra. Galicia: Decreto 269/2008, de 6 de noviembre, por el que se aprueba el Reglamento de Asistencia Jurídica Gratuita de Galicia (DOG, núm. 242, de 15 de diciembre de 2008); Cantabria: Decreto 86/2008, de 11 de septiembre, de Asistencia Jurídica Gratuita (BOC, núm. 27, de 14 de octubre de 2008); Andalucía: Decreto 67/2008, de 26 de febrero, por el que se aprueba el Reglamento de Asistencia Jurídica Gratuita en el ámbito de la Comunidad Autónoma de Andalucía (BOJA, núm. 44, de 4 de marzo de 2008), y Orden de 9 de marzo de 2009, por la que se aprueban los módulos y bases de compensación económica de los servicios de asistencia jurídica gratuita en el turno de oficio (BOJA, núm. 66, de 6 de abril de 2009); Madrid: Decreto 86/2003, de 19 de junio, por el que se regula la asistencia jurídica gratuita en el ámbito de la Comunidad de Madrid (BOCM, núm 148, de 24 de junio de 2003); Cataluña: Decreto 252/1996, de 5 de julio, de creación de las comisiones de asistencia jurídica gratuita, de regulación del procedimiento para el reconocimiento del derecho de asistencia jurídica gratuita y de la subvención para las actuaciones profesionales de los abogados y procuradores (DOGC, núm. 2228, de 10 de julio de 1996); Valencia: 
a la asistencia jurídica gratuita es, por tanto, complemento indefectible del derecho a la tutela judicial efectiva. De este modo, al establecerse como un derecho, las Administraciones Públicas deben asumir los costes del litigo en el caso de que los sujetos no dispongan de un nivel de renta suficiente y que es establecido por la ley.

El término «gratuita», como siempre en el caso de la provisión de determinados bienes por parte del sector público, es engañoso. El que la justicia sea gratuita no supone que no tenga costes, sino que éstos deben ser sufragados por otro agente diferente al que recibe los servicios, en este caso la Administración Pública, que financia sus recursos a través, principalmente, de los impuestos.

En España tienen derecho al beneficio de justicia gratuita los ciudadanos españoles y extranjeros residentes en España, los nacionales de Estados miembros de la UE, las asociaciones de utilidad pública y las fundaciones. Para obtener el derecho de justicia gratuita es necesario cumplir una serie de requisitos económicos. En el caso de las personas físicas es necesario, entre otros requisitos, que los recursos e ingresos de la unidad familiar no superen el doble del IPREM (Indicador Público de Renta de Efectos Múltiples). La evolución de las cuantías mensuales del IPREM en los últimos años aparece reflejada el en gráfico 1.

No obstante, si los solicitantes superan estas cuantías, y hasta un límite del cuádruplo del IPREM ${ }^{4}$, es posible que reciban excepcionalmente el beneficio de justicia gratuita si así lo estima oportuno una Comisión de Justicia Gratuita.

Una vez reconocido el derecho a la asistencia gratuita, el beneficiario recibe sin coste alguno para él una serie de prestaciones entre las que se incluyen los servicios profesionales de abogados, procuradores, peritos y resto de personal técnico que participa en el proceso. El derecho incluye,

Decreto 29/2001, de 30 de enero, de Asistencia Jurídica Gratuita (DOCV, núm. 3930, de 1 de febrero de 2001); Canarias: Decreto 57/1998, de 28 de abril, por el que se regulan la composición y el funcionamiento de las Comisiones de Asistencia Jurídica Gratuita de Canarias, así como el procedimiento para el reconocimiento de la misma (BOC, núm. 59, de 15 de mayo de 1998); Asturias: Decreto 273/2007, de 28 de noviembre, por el que se aprueba el Reglamento de Asistencia Jurídica Gratuita en el Principado de Asturias (BOPA, núm. 294, de 20 de diciembre de 2007); Aragón: Orden de 2 de enero de 2008, del Departamento de Política Territorial, Justicia e Interior, por la que se constituyen las Comisiones Provinciales de Asistencia Jurídica Gratuita de Zaragoza, Huesca y Teruel ( $B O A$, núm. 8, de 21 de enero de 2008); Navarra: Decreto Foral 80/2001, de 9 de abril, por el que se aprueba el Reglamento de Asistencia Jurídica Gratuita aplicable en la Comunidad Foral de Navarra (BON, núm. 57, de 9 de mayo de 2001) (Decreto Foral 707/2003, de 22 de diciembre).

${ }_{4} 29.820$ euros anuales en 2012. 
GRÁFICO 1

Evolución IPREM requerido (euros al mes), 2006-2012

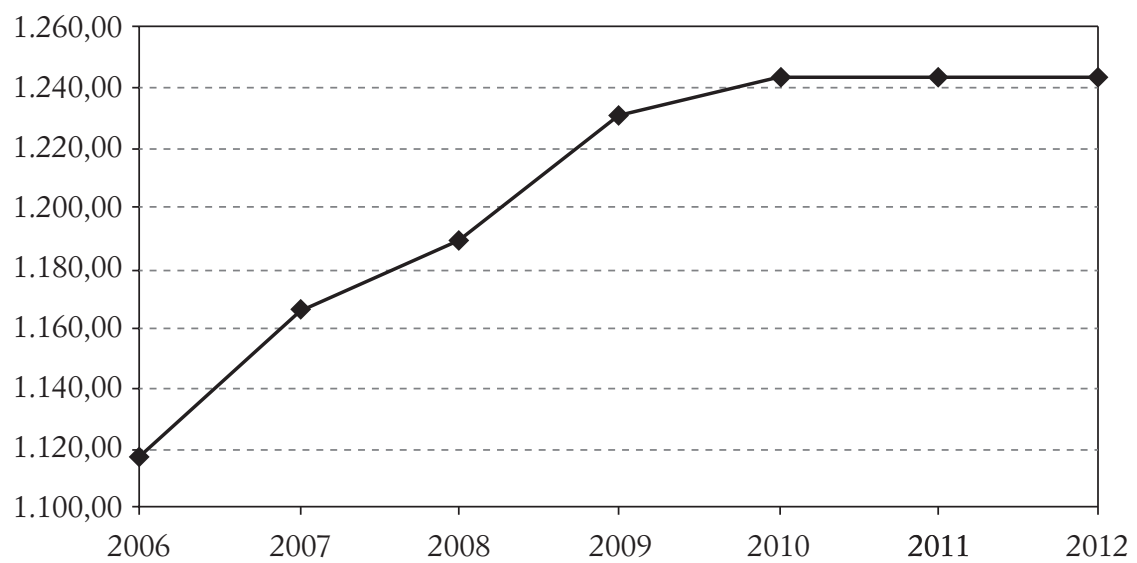

Fuente: elaboración propia.

además, la exención del pago de depósitos; la inserción gratuita de anuncios o edictos; la obtención gratuita de copias, testimonios, instrumentos y actas notariales, y la reducción de derechos arancelarios ${ }^{5}$. Estas prestaciones se extienden a todos los trámites e incidencias de una misma instancia y se mantienen para la interposición y sucesivos trámites de los posibles recursos $^{6}$. Esto no significa que el asesoramiento jurídico no se dé fuera del procedimiento judicial, ya que en nuestro ordenamiento la asistencia jurídica gratuita también incluye asesoramiento y orientación previos al proceso.

\section{Justificación}

No todos los ciudadanos tienen derecho a la justicia gratuita. Sólo aquellas familias con bajos niveles de renta pueden disfrutar de tal derecho. Se configura, por tanto, como un elemento de carácter redistributivo cuyo objetivo es permitir que determinados ciudadanos que carecen de recursos económicos puedan acceder a un derecho considerado fundamental. Esto justifica la intervención del sector público no sólo como ofe-

5 Art. 6 de la Ley 1/1996, de 10 de enero, de Asistencia Jurídica Gratuita.

${ }^{6}$ Art. 7 de la Ley 1/1996, de 10 de enero, de Asistencia Jurídica Gratuita. 
rente de los servicios, sino financiando éstos directamente a determinados sujetos que cumplan las condiciones requeridas; las Administraciones Públicas, de este modo, tienen la obligación de garantizar un derecho considerado como un servicio público de carácter básico.

Hay, sin embargo, otros argumentos de carácter económico que justifican el derecho a la justicia gratuita. Cuando un consumidor compra (consume) un bien recibe un beneficio privado por el que paga un precio de mercado sin que un tercero ajeno a la relación entre el vendedor y el comprador se beneficie del consumo de ese bien. Sin embargo, hay ocasiones en las que el consumo de un bien genera un beneficio en un tercero que no participa ni en la producción o venta del bien ni en su consumo. Hay, en este caso, lo que se denomina un beneficio público para distinguirlo del beneficio privado que recibe el consumidor que directamente paga y consume el bien. Pues bien, se dice que el beneficio público que se obtiene del consumo de los servicios de justicia es superior al beneficio privado. Y esto es así porque en el primero hay que incluir, además del beneficio privado, una serie de beneficios externos denominados externalidades positivas. Puede decirse que el consumo de un servicio genera una externalidad positiva cuando dicho consumo afecta a terceros que no participan ni en la oferta (venta o provisión) ni en la demanda (consumo) de ese servicio y esos efectos no se reflejan en el precio de mercado del servicio. La presencia de externalidades en la provisión de los servicios de justicia tiene como efecto más inmediato que la demanda que representa el valor social de los servicios sea superior a la demanda que refleja el beneficio privado de los mismos, ya que el valor social (beneficio social) incluye el beneficio privado y la externalidad positiva. Como resultado de esto, la cantidad de mercado del número de resoluciones judiciales, es decir, aquella en la que no se tienen en cuenta los beneficios externos asociados a los servicios de justicia, es inferior a la cantidad óptima desde el punto de vista social.

Fundamentalmente hay dos efectos positivos externos asociados a la provisión de los servicios de justicia: en primer lugar, la disuasión, y en segundo, la formación de precedentes jurídicos y el esclarecimiento del derecho. El primer efecto externo, la disuasión, se da porque los ciudadanos tienen la certeza de que si cometen actos ilícitos pueden acabar ante los tribunales y ser sancionados. Esta certeza, en función de determinadas circunstancias ${ }^{7}$, operará como elemento disuasorio a la hora de cometer un acto contrario

\footnotetext{
${ }^{7}$ Entre otras, aversión al riesgo, cuantía de la sanción, probabilidad de ser sancionado, beneficios por cometer el delito, etcétera.
} 
a las normas que puede perjudicar a otro miembro de la sociedad, lo que beneficia a ésta en su conjunto. El segundo efecto, la formación de precedentes jurídicos y el esclarecimiento del derecho, sirve para orientar el comportamiento de los litigantes y del conjunto de la población.

La presencia de estas externalidades positivas, tal y como se ha mencionado, tiene como efecto principal que los beneficios sociales derivados de la provisión de los servicios de justicia sean mayores que los beneficios privados. Consecuencia inmediata de esto último es que la cantidad óptima de resoluciones judiciales (litigios) sea mayor que la que genera el mercado por sí solo. Una manera de conseguir la cantidad óptima de litigios desde el punto de vista social es aumentar la demanda que refleja el valor privado de resoluciones judiciales hasta que iguale a la demanda que refleja el valor social. Esto puede conseguirse, entre otras medidas, subvencionando el acceso a la justicia a través del beneficio de justicia gratuita.

\section{LA JUSTICIA GRATUITA EN LA UNIÓN EUROPEA}

En la Unión Europea el acceso a los servicios de justicia se configura como un derecho, reconocido incluso constitucionalmente por algunos de sus países miembros ${ }^{8}$. El art. 47 de la Carta de los Derechos Fundamentales de la Unión Europea ${ }^{9}$, firmada en Niza el 7 de diciembre de 2000, establece que «se prestará asistencia jurídica gratuita a quienes no dispongan de recursos suficientes siempre y cuando dicha asistencia sea necesaria para garantizar la efectividad del acceso a la justicia». El Convenio Europeo para la Protección de los Derechos Humanos establece en su art. 6, párrafo 3, apartado c), el derecho de todo acusado a poder ser asistido gratuitamente por un abogado de oficio «si carece de medios para pagarlo». Así, todos los países miembros de la Unión Europea cuentan con un sistema nacional de justicia gratuita, pero en la práctica éstos difieren sustancialmente. No existe uniformidad ni en la organización ni en la gestión de los sistemas; hay divergencias en el contenido del derecho

\footnotetext{
8 Así, por ejemplo, la Constitución de Italia de 1947, en su art. 24, establece que: «Se garantizan a los desprovistos de recursos económicos, mediante las instituciones adecuadas, los medios para demandar y defenderse ante cualquier jurisdicción». La Constitución de Portugal, en su art. 20.1, proclama que: «Se garantiza a todos el acceso al derecho y a los tribunales para defender sus derechos e intereses protegidos legalmente, no pudiendo ser denegada la justicia por insuficiencia de medios económicos».

9 Diario Oficial, núm. C 364, de 18 de diciembre de 2000 pp. 0001-0022.
} 
y en los requisitos de acceso. Como ejemplo podemos analizar brevemente el casos de cuatro países de la UE: Bélgica, Alemania, Francia e Inglaterra, en los que se pueden apreciar las divergencias mencionadas, tanto en el contenido de la ayuda como en el umbral de ingresos mínimos requeridos para acceder a dicha ayuda.

\section{Bélgica}

La asistencia jurídica en Bélgica se compone de dos sistema aplicables en la jurisdicción civil y en la penal: la ayuda jurídica (de primera y de segunda línea) y la asistencia judicial. La ayuda jurídica de primera línea es una asistencia fuera del procedimiento judicial destinado a todas las personas físicas y jurídicas. Esta primera ayuda tiene un precio casi simbólico, salvo que el justiciable demuestre que carece de recursos económicos, en cuyo caso es gratis. La ayuda jurídica de segunda línea sólo está a disposición de las personas físicas que demuestren que no disponen de recursos suficientes, y consiste en un dictamen jurídico detallado o la asistencia jurídica dentro o fuera de un procedimiento, incluyendo la representación de un letrado. Por último, la asistencia judicial consiste en la exención del pago de una serie de gastos relacionados con un procedimiento judicial o extrajudicial ${ }^{10}$, y pueden beneficiarse de ella tanto los ciudadanos belgas como los extranjeros que cumplan determinados requisitos, siempre y cuando acrediten insuficiencia de recursos.

En cuanto al umbral de ingresos mínimos, el sistema distingue entre personas solas y unidad familiar; ambos pueden recibir una ayuda total o parcial. La ayuda total para personas solas se concede si éstas acreditan una renta mensual neta inferior a 907 euros $^{11}$; si se trata de personas con otras a su cargo o cónyuges o similares, la renta familiar neta debe ser inferior a 1.165 euros, que es el mínimo inembargable. En cuanto a la ayuda parcial, si se trata de personas solas deben acreditar que reciben una renta mensual neta de entre 907 y 1.165 euros; en el caso de personas con hijos a su cargo y cohabitantes, la renta neta debe encontrarse entre 1.165 y 1.423 euros.

\footnotetext{
${ }^{10}$ Gastos de timbre, registro, secretaría, expedición, etcétera.

11 Cuantías actualizadas a 1 de septiembre de 2011.
} 


\section{Alemania}

En Alemania el sistema de justicia gratuita distingue entre una ayuda que incluye el asesoramiento y, en su caso, la representación (Beratungshil$f e$ ), y una ayuda que cubre las costas (Prozesskostenhilfe). El primer tipo de ayuda se concede para asuntos de orden civil, administrativo, constitucional y laboral. En el orden penal y en el ámbito de las sanciones de tipo administrativo sólo se concede el asesoramiento. La ayuda en las costas se concede para las jurisdicciones civil, administrativa, fiscal y laboral.

Pueden acogerse al beneficio de justicia gratuita aquellas personas consideradas «indigentes» (Bedürftigkeit), es decir, que carezcan de los medios necesarios debido a su situación personal o económica, siempre y cuando no exista ninguna alternativa aceptable. Además, en el caso de la ayuda para las cosas es necesario que el tribunal que decide la concesión considere que el ejercicio de la acción tiene posibilidades de éxito.

\section{Inglaterra y País de Gales}

En Inglaterra y Gales la asistencia jurídica gratuita se ofrece en materia civil, existiendo cinco niveles de ayuda en función de la evaluación de la solicitud: Legal Help (asistencia jurídica), que consiste en un asesoramiento y ayuda inicial; Help at Court (asistencia ante los tribunales), en la que un abogado presta asistencia ante los tribunales pero sin una representación formal; Family Mediation (mediación familiar), mediación en conflictos familares; Family belp (ayuda familiar), consistente en prestar ayuda en negociaciones o en la obtención de órdenes o providencias judiciales relacionadas con disputas familiares, y Legal Representation (representación por abogado y procurador), que abarca la representación legal si el justiciable pretende iniciar un proceso.

Para poder beneficiarse de estas ayudas se tiene en cuenta tanto la renta disponible por el solicitante y su pareja ${ }^{12}$ como el capital disponible. En octubre de 2010 un sujeto podía acogerse a este sistema de ayuda si la renta bruta mensual (renta antes de impuestos) no superaba las 2.657 libras (3.322,91 euros) el mes anterior a la petición de ayuda y tenía un

12 Salvo que la relación haya finalizado o sea parte contraria en el caso. 
capital disponible inferior a 8.000 libras $(10.005,00 \text { euros })^{13}$. A pesar de reunir ambas condiciones, la solicitud debe someterse a una evaluación por parte de una comisión antes de aprobar la financiación.

\section{Francia}

En Francia, el sistema de asistencia jurídica gratuita comprende, en primer lugar, l'aide juridictionnelle (asistencia jurídica), que supone una ayuda financiera para la celebración de un juicio en cualquier jurisdicción; en segundo lugar, l'aide à l'intervention de l'avocat (asistencia para la representación del abogado), en procesos penales y asuntos penitenciarios, y, por último, l'accès au droit (el acceso al derecho), que incluye información, orientación y consulta jurídica gratuita.

Para beneficiarse de la ayuda jurídica es necesario que la media de los recursos mensuales de cualquier naturaleza del año precedente no supere los siguientes importes ${ }^{14}$ : 929 euros para una asistencia jurídica total y 1.393 euros para una ayuda jurídica parcial. Estas cantidades se aprueban cada año por ley y varían en función del número de personas a cargo del solicitante.

En lo referente al gasto público destinado a la asistencia jurídica también existen notables diferencias. Según los datos del Consejo de Europa, los países de la UE que más destinan a asistencia gratuita son, con diferencia, Inglaterra y Gales, cuyos datos se ofrecen conjuntamente, que en 2008 destinaron 1.878 millones de euros a este concepto, lo que supuso un gasto por habitante de 34,5 euros y un gasto por caso de 1.131 euros. La cantidad total presupuestada está muy alejada del resto de países, incluso después del descenso experimentado en el presupuesto de 2008 con respecto al de 2006, año en el que Inglaterra y Gales destinaron a la asistencia jurídica gratuita 3.020 millones de euros, lo que ha supuesto un descenso de aproximadamente el 40 por 100 (véase cuadro 3). No obstante este notable descenso, que también puede observarse en el resto del Reino Unido (Irlanda del Norte y, sobre todo, Escocia), sigue habiendo mucha diferencia con otros países como Alemania, que en 2006 destinó 557 millones de euros, o los Países Bajos, que en 2008 aprobaron un presupuesto de 419 millones de euros, siendo estos dos países los que, exceptuando Inglaterra y Gales, más destinan a la asistencia jurídica gratuita.

\footnotetext{
13 Estas cantidades pueden variar en función del número de hijos a cargo del solicitante.

${ }^{14}$ Cuantías en marzo de 2012.
} 


\section{CuAdro 1}

Presupuesto público anual destinado a la asistencia letrada y gasto presupuestario destinado a asistencia jurídica gratuita por caso en la Unión Europea en 2008 (en miles de euros)

\begin{tabular}{|c|c|c|}
\hline País & $\begin{array}{c}\text { Presupuesto público anual } \\
\text { destinado a la asistencia letrada }\end{array}$ & $\begin{array}{c}\text { Gasto presupuestario destinado } \\
\text { a asistencia jurídica gratuita por caso }\end{array}$ \\
\hline Austria & 18.400 .000 & - \\
\hline Bélgica & 60.277 .000 & 397 \\
\hline Bulgaria & 4.850 .000 & 113 \\
\hline República Checa & 25.995 .515 & 一 \\
\hline Dinamarca & 76.433 .980 & - \\
\hline Estonia & 2.934 .624 & 84 \\
\hline Francia & 314.445 .526 & 353 \\
\hline Grecia & 2.000 .000 & 一 \\
\hline Hungría & 319.765 & 7 \\
\hline Irlanda & 89.900 .000 & 1.432 \\
\hline Italia & 115.938 .469 & 787 \\
\hline Letonia & 1.087 .491 & - \\
\hline Lituania & 4.129 .000 & 94 \\
\hline Luxemburgo & 2.600 .000 & 714 \\
\hline Países Bajos & 419.248 .000 & 1.029 \\
\hline Polonia & 22.403 .000 & - \\
\hline Portugal & 36.432 .072 & 331 \\
\hline Rumania & 4.376 .694 & 30 \\
\hline Eslovaquia & 901.547 & - \\
\hline Eslovenia & 2.821 .428 & 431 \\
\hline España & 219.707.018 & 349 \\
\hline Suecia & 142.633 .089 & 一 \\
\hline Inglaterra y Gales & 1.878 .704 .340 & 1.131 \\
\hline Irlanda del Norte & 87.000 .000 & 1.021 \\
\hline Escocia & 15.000 .000 & 537 \\
\hline Media & 141.941 .542 & 520 \\
\hline Mediana & 22.403 .000 & 397 \\
\hline Mínimo & 319.765 & 7 \\
\hline Máximo & 1.878 .704 .340 & 1.432 \\
\hline
\end{tabular}

Fuente: European Commission for the Efficiency of Justice (CEPEJ), European judicial systems 2010 (data 2008): Efficiency and quality of justice. 
GRÁFICO 2

Presupuesto público anual destinado a la asistencia letrada en el año 2008 (euros)

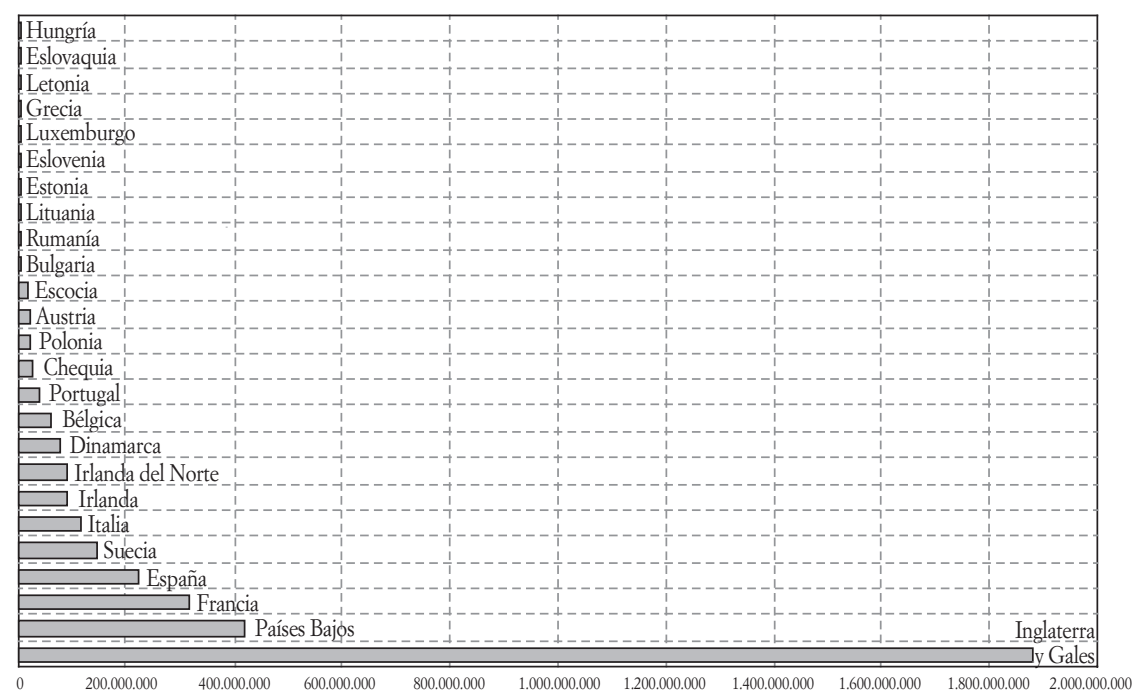

Por otro lado, es Irlanda del Norte el país que más presupuesto destina por habitante (49,5 euros), dedicando un total de 87 millones de euros a la asistencia gratuita. El país que menos presupuesto destina a este concepto es Hungría, que en 2008 tan sólo dedicó a asistencia jurídica gratuita 319.765 euros, 7 euros por caso y 0,03 euros por habitante.

La media del presupuesto de todos los países de la UE, exceptuando a Alemania, destinado a asistencia jurídica gratuita fue en 2008 de 141.941.542 euros, lo que sitúa a España por encima de la media. Sin embargo, nuestro país está por debajo de la media europea en lo que se refiere al gasto por caso, ya que la media de gasto presupuestado destinado a la asistencia jurídica gratuita en España ese año fue de 349 euros por caso cuando la media de la UE fue de 520 euros. También España está por debajo de la media en lo que se refiere a presupuesto destinado a asistencia jurídica gratuita por habitante. La media europea se sitúa en 7,2 euros por habitante y en España se destinan 4,9 euros.

En cuanto a la evolución del presupuesto, en la mayoría de los países se ha observado una tendencia al aumento del gasto público destinado a la asistencia jurídica gratuita salvo en el Reino Unido, Luxemburgo, Rumania y Eslovaquia. Como ya se ha mencionado, los descensos más significativos son los de Inglaterra y Gales, y Escocia. 
GRÁFICO 3

Gasto presupuestario destinado a asistencia jurídica gratuita por caso en el año 2008 (euros)

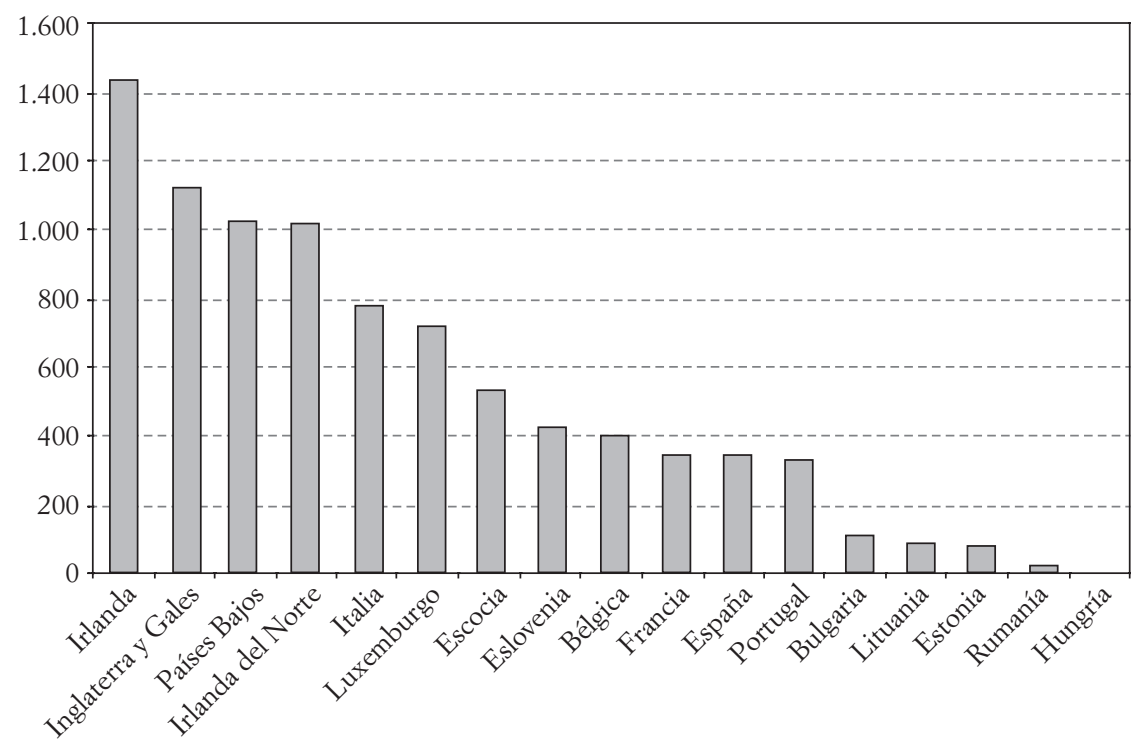

CuAdro 2

Evolución del presupuesto público anual destinado a la asistencia letrada en la UE (en miles de euros)

\begin{tabular}{|l|r|r|r|r|}
\hline \multicolumn{1}{|c|}{ País } & \multicolumn{1}{c|}{2002} & \multicolumn{1}{c|}{2004} & \multicolumn{1}{c|}{2006} & \multicolumn{1}{c|}{2008} \\
\hline Austria & - & 24.100 .000 & 17.700 .000 & 18.400 .000 \\
\hline Alemania & 462.000 .000 & 468.400 .000 & 557.000 .000 & - \\
\hline Bélgica & 40.225 .000 & 30.750 .000 & 43.137 .000 & 60.277 .000 \\
\hline Bulgaria & 1.132 .581 & 1.571 .358 & 1.804 .100 & 4.850 .000 \\
\hline República Checa & - & 12.273 .022 & 15.672 .575 & 25.995 .515 \\
\hline Dinamarca & 38.935 .860 & 3.200 .000 & 2.869 .941 & 76.433 .980 \\
\hline Estonia & - & 1.700 .000 & 2.567 .320 & 2.934 .624 \\
\hline Francia & 279.000 .000 & 291.200 .000 & 303.000 .000 & 314.445 .526 \\
\hline Grecia & - & 724.187 & 1.700 .000 & 2.000 .000 \\
\hline
\end{tabular}




\begin{tabular}{|c|c|c|c|c|}
\hline País & 2002 & 2004 & 2006 & 2008 \\
\hline Hungría & - & 851.333 & 198.981 & 319.765 \\
\hline Irlanda & 54.700 .000 & 47.649 .000 & 63.600 .000 & 89.900 .000 \\
\hline Italia & 44.735 .178 & 66.030 .256 & 86.562 .704 & 115.938 .469 \\
\hline Letonia & 689.400 & 653.490 & 1.072 .771 & 1.087 .491 \\
\hline Lituania & 一 & 一 & 3.226 .245 & 4.129 .000 \\
\hline Luxemburgo & 一 & 2.574 .828 & 2.949 .983 & 2.600 .000 \\
\hline Países Bajos & 202.627 .400 & 378.358 .000 & 344.666 .748 & 419.248 .000 \\
\hline Polonia & - & 16.775 .566 & 21.724 .000 & 22.403 .000 \\
\hline Portugal & 一 & 27.632 .424 & 35.829 .192 & 36.432 .072 \\
\hline Rumania & 一 & 1.810 .732 & 6.065 .759 & 4.376 .694 \\
\hline Eslovaquia & 一 & 1.967 .026 & 2.779 .410 & 901.547 \\
\hline Eslovenia & 一 & 一 & 1.858 .859 & 2.821 .428 \\
\hline España & 95.864 .422 & 119.055 .984 & 167.331 .526 & 219.707.018 \\
\hline Suecia & 103.595 .240 & 95.455 .900 & 150.764 .128 & 142.633 .089 \\
\hline Inglaterra y Gales & 2.800 .000 .000 & 3.070 .000 .000 & 3.020 .104 .244 & 1.878 .704 .340 \\
\hline Irlanda del Norte & 31.560 .000 & 93.630 .000 & 95.772 .010 & 87.000 .000 \\
\hline Escocia & 218.200 .000 & 216.000 .000 & 239.947 .427 & 15.000 .000 \\
\hline
\end{tabular}

Fuente: European Commission for the Efficiency of Justice (CEPEJ).

\section{LA JUSTICIA GRATUITA EN ESPAÑA}

El análisis del gasto que realizan las Administraciones Públicas relacionado con la justicia gratuita no es sencillo. Por un lado hay dificultades a la hora de evaluar el verdadero coste del servicio, ya que hay que distinguir entre el presupuesto aprobado y el presupuesto realmente ejecutado. Y una vez conocido el presupuesto ejecutado es difícil agrupar los diferentes conceptos a los que se ha destinado, ya que hay discrepancias en los datos ofrecidos por los colegios de abogados (importes certificados), otros conceptos asociados a los costes de la justicia gratuita ${ }^{15} \mathrm{y}$ las cifras globales.

${ }^{15}$ Como son los derechos de los procuradores, la publicación de edictos, derechos de peritos, derechos arancelarios, etcétera. 
La fuente fundamental para conocer el verdadero coste del sistema lo ofrecen las certificaciones que presentan el Consejo General de la Abogacía (en adelante CGAE) y el Consejo General de Procuradores de los Tribunales (en adelante CGPT) al Ministerio de Justicia y que contienen el número y clase de actuaciones realizadas por los abogados y procuradores de cada colegio durante un trimestre, incluyendo una justificación del coste económico de la actuaciones. No es la única fuente, pero sí la de mayor relevancia en cuanto al volumen del coste. Cada servicio prestado por los abogados y procuradores tiene un coste establecido por unos baremos aprobados por la Administración Pública. En el sistema de cobro participan varios agentes: en primer lugar, los abogados y procuradores que han prestado el servicio deben justificar documentalmente la intervención profesional ante sus respectivos colegios, documentación que debe ser verificada por éstos. Los colegios deben enviar la documentación al CGAE y CGPT que son los encargados de distribuir trimestralmente el importe de las subvenciones. Así, el mes natural siguiente al de la finalización de cada trimestre el CGAE y el CGPE remiten al Ministerio de Justicia la mencionada certificación. El Ministerio de Justicia efectúa el libramiento de los certificados que correspondan a través del Tesoro Público pagando a los consejos generales, que a su vez pagan a los colegios correspondientes, los cuales retribuyen el importe de las subvenciones a los abogados y procuradores (véase figura 1).

La fuente para conocer el total de importes certificados por servicios de asistencia jurídica gratuita son los Colegios de Abogados de España. Para realizar un análisis detallado del coste de estos servicios habría que comparar los importes certificados con los gastos presupuestados aprobados y ejecutados en cada territorio. Y esto es una tarea extremadamente difícil no sólo porque las fuentes en muchos casos no coinciden en sus cifras, sino porque es posible que a efectos presupuestarios no sólo estos importes formen parte de los gastos relacionados con la justicia gratuita. Conocer todos los conceptos requeriría un estudio pormenorizado del sistema, profundizar en las fuentes estadísticas de cada Comunidad y realizar un análisis comparativo. Pero una primera aproximación se puede llevar a cabo analizando los Presupuestos Generales del Estado (en adelante PGE) que financian los servicios de asistencia jurídica gratuita en territorios donde no se ha transferido la competencia sobre esta materia.

Los PGE clasifican los gastos atendiendo a tres criterios: la finalidad u objetivo (clasificación por programas), la organización del sector público estatal (clasificación orgánica) y la naturaleza económica de los gastos (clasificación económica). 
FigURA 1

Procedimiento de cobro de las certificaciones por actuaciones de justicia gratuita

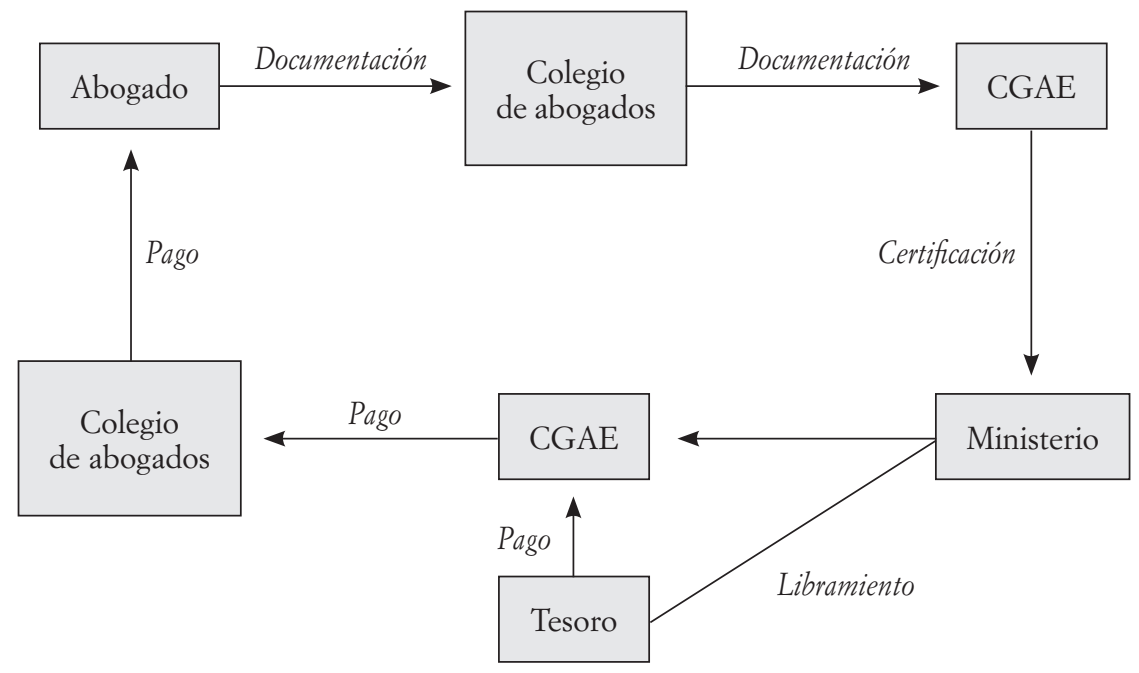

La clasificación por programas permite conocer en qué se gasta el dinero del contribuyente el sector público, es decir, los programas de gasto del sector público. Estos programas se agrupan formando grupos de programa y éstos a su vez se integran en políticas de gasto. Las políticas de gasto se estructuran en áreas de gasto, que son cinco: servicios públicos básicos (entre los que se encuentra la Justicia), actuaciones de protección y promoción social, producción de bienes públicos de carácter preferente, actuaciones de carácter económico y actuaciones de carácter general. De este modo, «Justicia» es una política de gasto incluida entre los denominados servicios públicos básicos, que incluye tres grupos de programas (véase figura 2):

1. Administración General de Justicia, formado por los programas «Dirección y Servicios Generales de Justicia», «Formación del Personal de la Administración de Justicia» y «Formación de la Carrera Fiscal».

2. Administración de Justicia, formado por el programa «Tribunales de Justicia y Ministerio Fiscal».

3. Registros, que incluye el programa «Registros vinculados con la Fe Pública». 
Presupuesto por programas y memoria de objetivos. Justicia

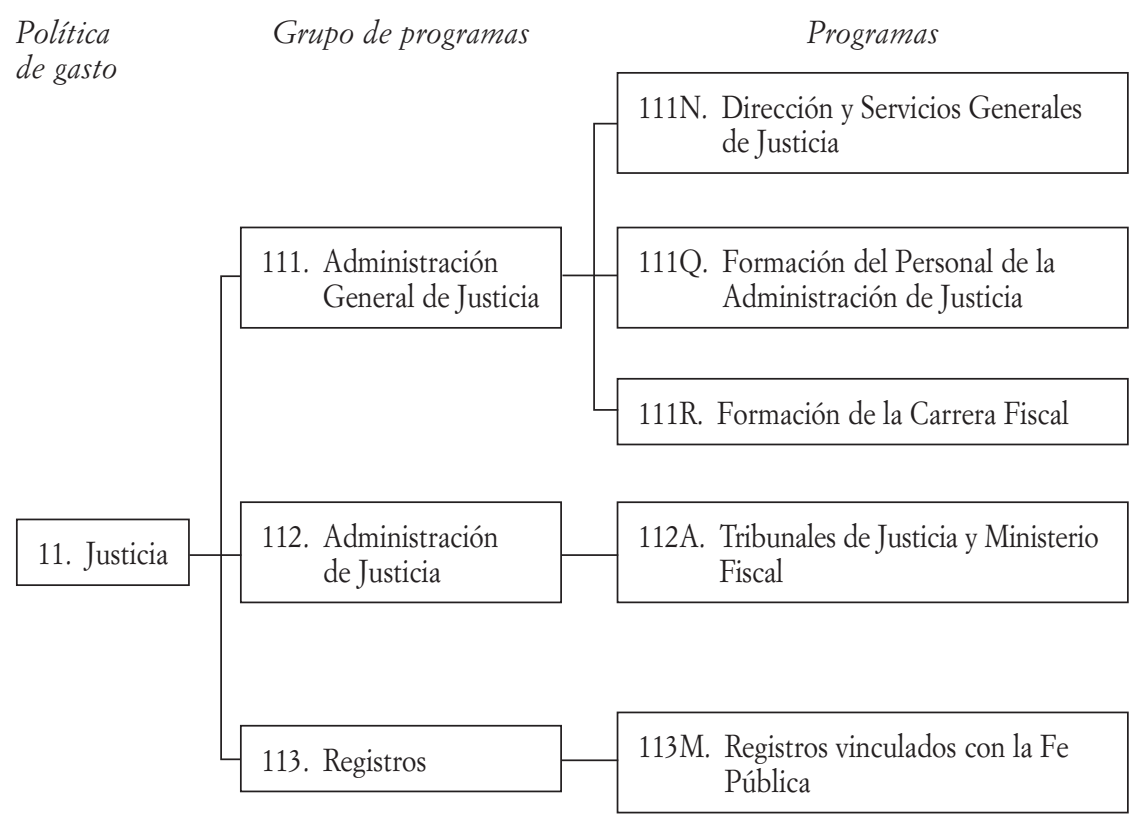

Fuente: PGE, presupuesto aprobado en 2011, Serie roja, Gastos, Presupuesto por programas y memoria de objetivos.

El Presupuesto de Justicia de 2011, incluido en el área Servicios Públicos Básicos, fue de 1.713,25 millones de euros. Hay, sin embargo, una dotación presupuestaria incluida en esta misma área de gasto denomina$\mathrm{da}$ «Seguridad ciudadana e instituciones penitenciarias» que puede incluir gastos relacionados con la asistencia jurídica gratuita. Por su parte, el Presupuesto consolidado de 2011 para la política de gasto «Justicia» fue de 1.819 millones de euros (Justicia homogeneizado).

Es dentro del programa «Tribunales de Justicia y Ministerio Fiscal» en el que se incluyen los gastos relacionados con la asistencia jurídica gratuita, en concreto las transferencias al CGAE y al CGPE, encargadas, como se ha señalado, de distribuir trimestralmente el importe de las subvenciones.

La clasificación económica de los Presupuestos permite conocer cómo se gasta y clasifica los gastos de cada sección (en el caso de Justicia, el 


\section{GRÁFICO 4}

Asistencia jurídica gratuita. Presupuestos Generales del Estado 2011 (miles de euros)

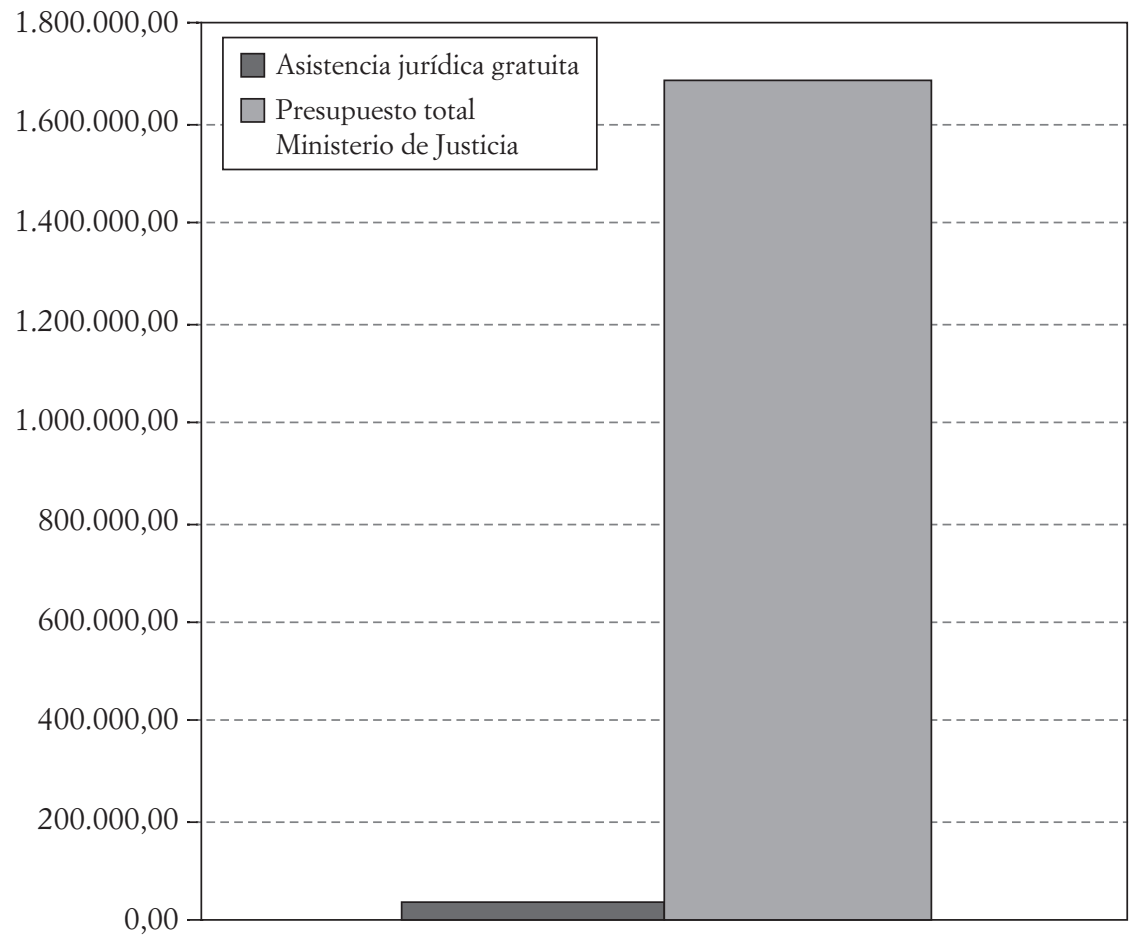

Fuente: elaboración propia a partir de datos de los PGE 2011.

Ministerio de Justicia) en capítulos ${ }^{16}$, que pueden clasificarse en artículos y éstos a su vez en conceptos y subconceptos. A estos efectos presupuestarios, los gastos relacionados con la asistencia jurídica gratuita están considerados como una transferencia corriente y se incluyen en el capítulo IV, «Transferencias corrientes», art. 46, «A familias e instituciones sin fines de lucro», conceptos 483, «Transferencias al CGAE», y 484, «Transferencias al CGPE».

De este modo, el total del gasto aprobado del presupuesto del Ministerio de Justicia incluido en los PGE de 2011 fue de 1.680.505.000,35 euros,

\footnotetext{
${ }^{16}$ Gastos de personal, gastos corrientes en bienes y servicios, gastos financieros, transferencias corrientes, inversiones reales, transferencias de capital y activos financieros, etcétera.
} 


\section{GRÁFICO 5}

Gasto en asistencia jurídica gratuita en relación al total de transferencias corrientes del presupuesto para el Ministerio de Justicia

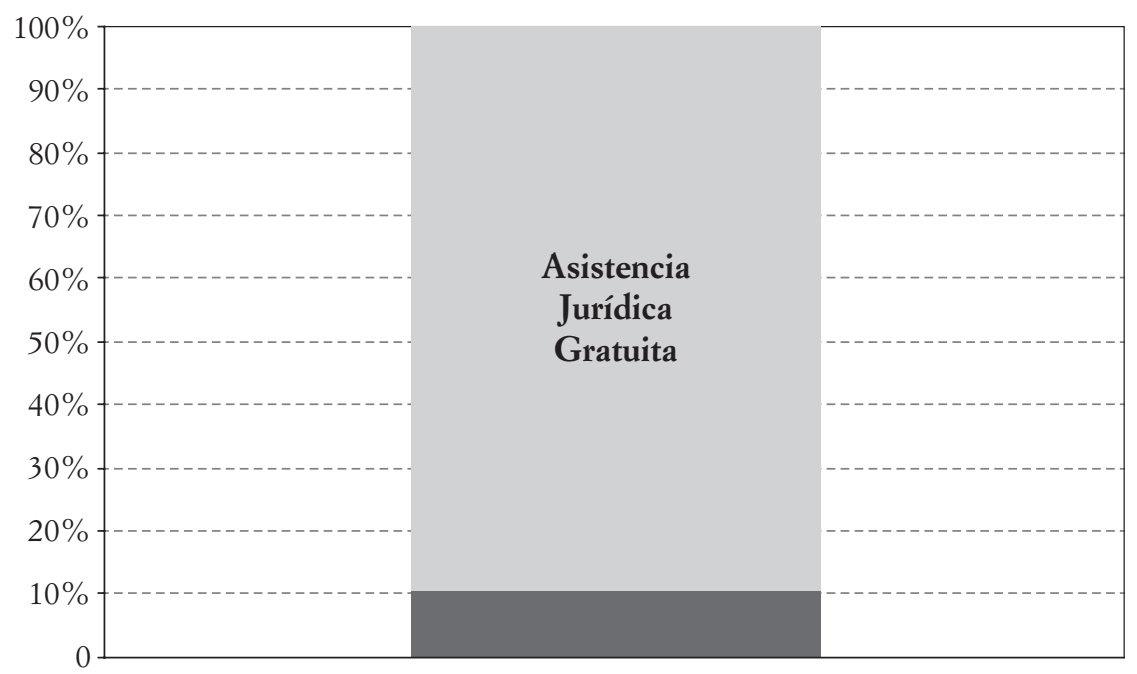

Fuente: elaboración propia a partir de datos de los PGE 2011.

de los cuales $35.550 .000,13$ euros (el 2,11 por 100) se destinaron a transferencias corrientes. Del total de las transferencias corrientes, 32.527.000,34 euros (es decir, el 91,49 por 100) fueron transferencias a familias e instituciones sin fines de lucro. Esta partida incluye la mayor parte de los gastos relacionados con la asistencia jurídica gratuita dividiendo el gasto en los dos conceptos siguientes:

1. Concepto 483: transferencias corrientes al CGAE como aportación del Estado para indemnizar a los abogados en los asuntos de asistencia jurídica gratuita: 29.541.000,52 euros.

2. Concepto 484: transferencias corrientes al CGPE como aportación del Estado para indemnizar a los procuradores en los asuntos de asistencia jurídica gratuita: 2.313.000,21 euros.

El total de transferencias presupuestadas y destinadas a justicia gratuita fue, por tanto, de 31.854.000,73 euros, lo que supone el 89,60 por $100 \mathrm{del}$ total de las transferencias corrientes del presupuesto del Ministerio de Justicia y el 1,89 por 100 del total del presupuesto aprobado de este departamento ministerial (véanse gráficos 4, 5 y 6). 


\section{GRÁFICO 6}

Transferencias corrientes del presupuesto aprobado para el Ministerio de Justicia en 2011

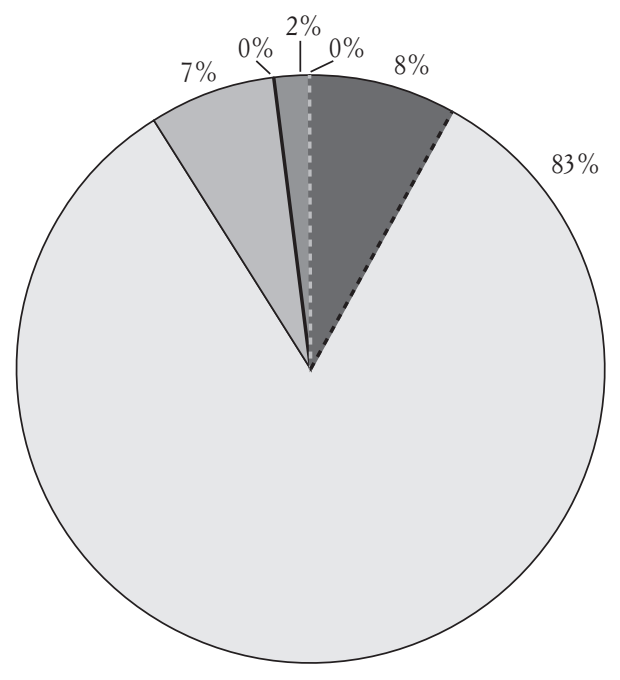

Subvención por gastos Juzgados de Paz

III Subvención Asociaciones de Secretarios Judiciales

$\square$ Al CGAE como aportación del Estado para indemnizar a los abogados en los asuntos de asistencia jurídica gratuita

Al CGPE como aportación del Estado para indemnizar a los procuradores en los asuntos de asistencia jurídica gratuita

- Subvenciones a Fundaciones Judiciales

$\square$ Al Consejo General de Colegios Oficiales de Psicólogos para asistencia psicológica a las víctimas

III Subvención Asociaciones de Médicos Forenses

Los datos de los presupuestos de los años anteriores son similares y no han experimentado variaciones sustanciales. En el cuadro 3 se presentan datos de la evolución de las transferencias corrientes al CGAE y al CGPE como aportación del Estado para indemnizar a los abogados y procuradores en los asuntos de asistencia jurídica gratuita.

Según el V Informe del Observatorio de la Justicia Gratuita del Consejo General de la Abogacía Española, en 2010 el total de importes certificados en territorios en los que no se han transferido las competencias en materia de justicia gratuita fue de 31.533 .106 euros $^{17}$. El total presupuestado para ese año fue, como se observa en el cuadro 4, de 34.887.000 euros. La discrepancia es evidente y previsible, ya que es imposible conocer con antelación el número de actuaciones que habrá durante el año ${ }^{18}$. De ahí que sea imprescindible acudir al presupuesto ejecutado.

${ }_{17}$ Incluye los siguientes servicios: turno de oficio, asistencia letrada al detenido, actuaciones por violencia de género y gastos en infraestructura.

${ }_{18}$ Más cercana a la cifra que ofrecen los importes certificados en 2010 es la cuantía presupuestada para el ejercicio siguiente (2011), lo que es en cierto modo lógico e indicativo de la relevancia de los importes certificados a la hora de conocer el coste del servicio. 


\section{CuAdro 3}

Evolución de las transferencias por asistencia jurídica gratuita en los PGE 2006-2011 (en miles de euros)

\begin{tabular}{|l|c|c|c|c|c|c|}
\hline & 2011 & \multicolumn{1}{|c|}{2010} & \multicolumn{1}{c|}{2009} & \multicolumn{1}{c|}{2008} & \multicolumn{1}{c|}{2007} & \multicolumn{1}{c|}{2006} \\
\hline Al CGAE & 29.541 .001 & 32.354 .000 & 28.664 .001 & 31.300 .001 & 30.237 .000 & 25.467 .000 \\
\hline Al CGPE & 2.313 .000 & 2.533 .000 & 2.233 .000 & 2.418 .001 & 2.077 .001 & 2.159 .000 \\
\hline TOTAL & 31.854 .001 & 34.887 .000 & 30.897 .001 & 33.718 .001 & 32.314 .001 & 27.626 .000 \\
\hline
\end{tabular}

Fuente: Presupuestos Generales del Estado.

La clasificación orgánica de los PGE permite conocer quién gasta y distingue entre centros gestores, secciones y servicios. En el caso de la Justicia, el centro gestor es el Estado y la sección el Ministerio de Justicia (Sección 13 en los PGE de 2011) que incluye los siguientes servicios:

1. Ministerio, Subsecretaría y Servicios Generales.

2. Secretaría General de Modernización y Relaciones con la Administración de Justicia.

3. Dirección General de los Registros y del Notariado.

4. Abogacía General de Estado-Dirección del Servicio Jurídico del Estado.

5. Secretaría General de Modernización y Relaciones con la Administración de Justicia-Fiscalía General del Estado.

Los gastos relacionados con la asistencia jurídica gratuita dependen del servicio 02, «Secretaría General de Modernización y Relaciones con la Administración de Justicia».

Por secciones, la ejecución y liquidación del presupuesto de Justicia correspondiente al ejercicio 2011 fue la siguiente:

\section{CuAdro 4}

Presupuesto de gastos (Justicia). Estado de ejecución del Presupuesto 2011 por secciones (miles de euros)

\begin{tabular}{|l|c|c|}
\hline & & $\begin{array}{c}\text { Porcentaje } \\
\text { de realización }\end{array}$ \\
\hline Créditos totales & 1.680 .644 & - \\
\hline Compromisos de gastos & 1.487 .066 & 88,48 \\
\hline Obligaciones reconocidas & 316.074 & 18,80 \\
\hline
\end{tabular}

Fuente: IGAE. 
Estos datos reflejan la discrepancia entre el presupuesto aprobado y el presupuesto ejecutado. Un estudio más detallado de la ejecución de los presupuestos permitiría conocer con detalle la correspondencia del presupuesto ejecutado con las certificaciones entregadas por los colegios. Sin embargo, no es tarea fácil porque en los presupuestos o partidas que financian las subvenciones a los servicios de justicia gratuita pueden intervenir no sólo el Estado o las Comunidades Autónomas, sino otros entes locales, como es el caso de los Ayuntamientos ${ }^{19}$. Sí es cierto que cada Administración autonómica se encarga del pago en su territorio, y allí donde esta competencia no corresponde a la Comunidad Autónoma el pago es efectuado por el Estado. En este sentido los datos del presupuesto ejecutado del Estado relacionados con la dotación para el funcionamiento del sistema de justicia gratuita disponibles son los siguientes:

\section{CuAdro 5}

Dotación de medios para el funcionamiento del sistema de justicia gratuita. Presupuesto ejecutado 2006-2009 (miles de euros)

\begin{tabular}{|l|c|c|c|c|}
\hline & 2009 & 2008 & 2007 & 2006 \\
\hline Presupuestado & $30.897,88$ & $33.719,20$ & $32.314,87$ & $29.241,88$ \\
\hline Ejecutado & $34.236,86$ & $28.931,44$ & $33.622,50$ & $33.229,75$ \\
\hline
\end{tabular}

Fuente: PGE 2006-2009, Serie roja, Gastos, Presupuesto por programas y memoria de objetivos de los programas del Ministerio de Justicia.

Comparando los datos del cuadro 3 con las dotaciones del cuadro 5 se observan discrepancias en las cifras: en los años 2006 y 2008 no coinciden las cantidades aprobadas por el Ministerio para el funcionamiento del sistema y el total del presupuesto aprobado y destinado a los colegios, coincidencia que sí se da en los años 2007 y 2009. Las causas de estas discrepancias requerirían un estudio más detallado.

${ }_{19}$ Así, y a modo de ejemplo, en la ejecución del Presupuesto General del Ayuntamiento de Madrid para 2010 hay una partida de 168.070 euros cuyo beneficiario es el Colegio de Abogados de Madrid y que tiene como objeto la «orientación jurídica en materia de extranjería y en supuestos de racismo, xenofobia, homofobia y transfobia». Para conocer si este tipo de partidas forman parte de los gastos relacionados con la asistencia jurídica gratuita haría falta un estudio más detallado de la cuestión. 
Por su parte, los Presupuestos Generales de las Comunidades Autónomas destinan una parte de sus transferencias corrientes a los servicios de asistencia jurídica gratuita. Como ejemplo ilustrativo puede mencionarse que en los Presupuestos Generales de la Comunidad de Madrid hay dos dotaciones presupuestarias destinadas al funcionamiento de los servicios del turno de oficio, asistencia letrada al detenido y asistencia jurídica gratuita. Se trata de los subconceptos 4456, «Transferencias corrientes a Colegios de Abogados», y 4457, «Transferencias corrientes a Colegios de Procuradores». En 2011 las cantidades presupuestadas fueron las siguientes:

\section{CuAdro 6}

Presupuesto público anual destinado a la asistencia letrada en la Comunidad de Madrid en 2011 (miles de euros)

\begin{tabular}{|l|r|}
\hline A Colegios de Abogados & 30.897 .400 \\
\hline A Colegios de Procuradores & 3.127 .520 \\
\hline Total asistencia jurídica gratuita & 34.024 .920 \\
\hline
\end{tabular}

Fuente: Presupuestos Generales de la CAM 2011.

\section{CONCLUSIONES}

Es difícil conocer el verdadero esfuerzo que realiza el sector público en la provisión de los servicios de justicia gratuita. La dispersión de los datos, la distribución de competencias y la ausencia de fuentes estadísticas específicas y uniformes dificultan considerablemente el estudio. No obstante la dificultad del análisis, los datos reflejan que nuestro país no está precisamente a la cabeza del servicio en lo que a gasto se refiere. La actual crisis económica, que ha tenido como consecuencia más notoria el recorte de los gastos destinados a determinados servicios públicos de carácter redistributivo, ha aumentado más si cabe la brecha con los países de nuestro entorno. Se hace, por tanto, necesario profundizar en el estudio para conocer el verdadero gasto de las Administraciones con el fin de compararlo con los beneficios sociales que el sistema genera en la población. 


\section{BIBLIOGRAFÍA}

Cabrillo, F., y Fitzpatrick, S., La economía de la Administración de Justicia, Cizur Menor, Civitas Thomson Reuters, 2011.

Pastor, S., jAh de la justicia! Política judicial y economía, Madrid, Civitas, 1993.

Comunidad Autónoma de Madrid, Presupuestos Generales de la CAM, Madrid, 2011.

Consejo General de la Abogacía Española, VI Informe del Observatorio de la Justicia Gratuita, Madrid, La Ley, 2012.

España, Presupuestos Generales del Estado, 2006.

- Presupuesto aprobado, Serie roja, Estado (anual).

- Ministerio de Hacienda y Administraciones Públicas, Intervención General de la Administración del Estado, Estadísticas de Ejecución del Presupuesto.

European Commission for the EfFiciency of Justice (CEPEJ), European judicial systems 2010 (data 2008): Efficiency and quality of justice. 